\title{
FAM225B Is a Prognostic lncRNA for Patients with Recurrent Glioblastoma
}

\author{
Junsheng Li $\mathbb{D}^{1,2,3,4,5}$ Qian Zhang, ${ }^{1,2,3,4,5}$ Peicong Ge, ${ }^{1,2,3,4,5}$ Chaofan Zeng $\mathbb{D}^{1,2,3,4,5}$ \\ Fa Lin, ${ }^{1,2,3,4,5}$ Wen Wang $\mathbb{D}^{1,2,3,4,5}$ and Jizong Zhao $\mathbb{D}^{1,2,3,4,5,6}$ \\ ${ }^{1}$ Department of Neurosurgery, Beijing Tiantan Hospital, Capital Medical University, China \\ ${ }^{2}$ China National Clinical Research Center for Neurological Diseases, China \\ ${ }^{3}$ Center of Stroke, Beijing Institute for Brain Disorders, China \\ ${ }^{4}$ Beijing Key Laboratory of Translational Medicine for Cerebrovascular Disease, China \\ ${ }^{5}$ Beijing Translational Engineering Center for 3D Printer in Clinical Neuroscience, China \\ ${ }^{6}$ Savaid Medical School, University of the Chinese Academy of Sciences, China \\ Correspondence should be addressed to Wen Wang; wangwenttyy@126.com and Jizong Zhao; zhaojz205@163.com
}

Received 26 September 2020; Revised 22 October 2020; Accepted 29 October 2020; Published 23 November 2020

Academic Editor: Wen-Jun Tu

Copyright (C) 2020 Junsheng Li et al. This is an open access article distributed under the Creative Commons Attribution License, which permits unrestricted use, distribution, and reproduction in any medium, provided the original work is properly cited.

\begin{abstract}
Objective. The overall survival of patients with recurrent glioblastoma (rGBM) is quite different, so clinical outcome prediction is necessary to guide personalized clinical treatment for patients with rGBM. The expression level of lncRNA FAM225B was analyzed to determine its prognostic value in rGBMs. Methods. We collected 109 samples of Chinese Glioma Genome Atlas (CGGA) RNA sequencing dataset and divided into training set and validation set. Then, we analyzed the expression of FAM225B, clinical characteristics, and overall survival (OS) information. Kaplan-Meier survival analysis was used to estimate the OS distributions. The prognostic value of FAM225B in rGBMs was tested by univariate and multivariate Cox regression analyses. Moreover, we analyzed the biological processes and signaling pathways of FAM225B. Results. We found that FAM225B was upregulated in rGBMs $(P=0.0009)$. The expression of FAM225B increased with the grades of gliomas $(P<0.0001)$. The OS of rGBMs in the low-expression group was significantly longer than that in the high-expression group $(P=0.0041)$. Similar result was found in the training set $(P=0.0340)$ and verified in the validation set $(P=0.0292)$. In multivariate Cox regression analysis, FAM225B was identified to be an independent prognostic factor for rGBMs $(P=0.003)$. Biological process and KEGG pathway analyses implied FAM225B mainly played a functional role on transcription, regulation of transcription, cell migration, focal adhesion, etc. Conclusions. FAM225B is expected to be as a new prognostic biomarker for the identification of rGBM patients with poor outcome. And our study provided a potential therapeutic target for rGBMs.
\end{abstract}

\section{Introduction}

As the most common intracranial malignant tumor in adults $[1,2]$, glioblastoma (GBM) has a median overall survival (OS) of only 14.6 months [3]. Most GBMs will relapse after standard treatment such as surgical resection, radiotherapy, and chemotherapy [4-6]. In recurrent glioblastoma (rGBM) cases, the patients may lose the opportunity for surgery because of the invasion to the functional area by tumors [7]. And rGBMs are less sensitive to chemotherapy or radiotherapy than primary ones as well [8-10]. So progress in prognostic biomarker identification is required for person- alized treatment of patients with rGBM [11]. Further understanding the molecular factors of rGBMs will provide novel insights for the potential biological characteristics and elucidate the possible therapeutic targets.

Long noncoding RNAs (lncRNAs) refer to noncoding RNAs more than 200 nucleotides in length $[12,13]$ and are associated with a series of cellular process [14-16], such as epigenetic regulation and transcriptional regulation. The dysregulated $\operatorname{lncRNAs}$ can be used as prognostic factors for patients [17-20].

In this study, we collected 109 rGBM samples from the Chinese Glioma Genome Atlas (CGGA, http://www.cgga 
FAM225B

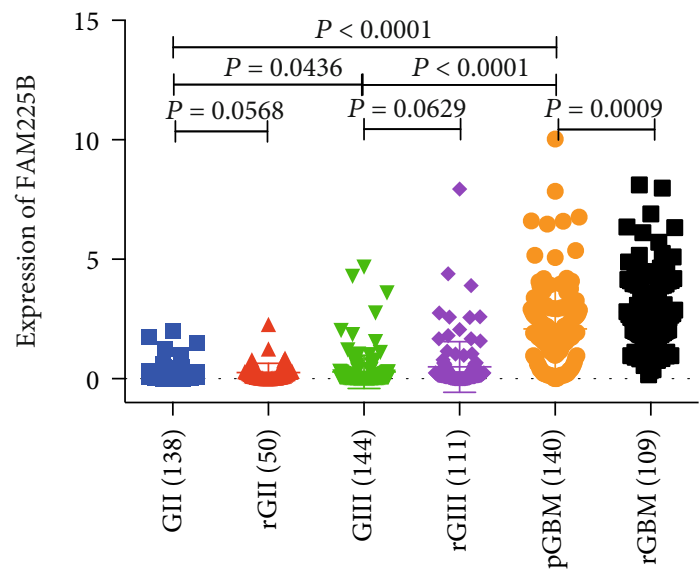

(a)
FAM225B (rGBM)

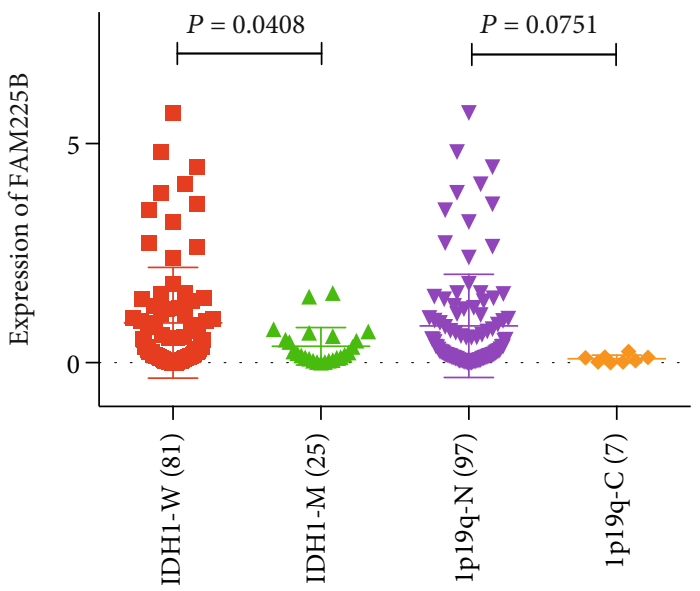

(b)

Figure 1: The expression pattern of FAM225B in gliomas: (a) FAM225B expression in different grades (grade II to grade IV) and types (primary or recurrent); (b) FAM225B expression in different IDH1 status and 1p/19q status.

.org.cn/) RNA sequencing dataset. We identified a new prognostic lncRNA FAM225B in rGBMs. We investigated the expression patterns of FAM $225 B$ and evaluated its prognostic value. The expression of FAM225B increased with the glioma grades, and it indicated the poor prognosis in rGBM patients. Therefore, FAM225B could be a prognostic indicator and a potential therapeutic target for rGBMs.

\section{Materials and Methods}

2.1. Patients and Datasets. Our study obtained 109 rGBM samples from the CGGA RNA sequencing dataset with expression data and clinical information [21,22]. All samples were initially diagnosed with primary glioblastoma (pGBM) and relapsed after standard treatment. The cases were randomly divided into training set and validation set without bias [23]. And the median relative expression of FAM225B was used as the cut-off value to divide the samples into a low-expression group and high-expression group. All these samples were diagnosed histologically by 2 neuropathologists according to the $2016 \mathrm{WHO}$ classification guideline of nervous system tumors. Our study was approved by the Ethics Committee of Beijing Tiantan Hospital.

2.2. Statistical and Bioinformatic Analysis. SPSS software (version 22; SPSS Inc., Chicago, IL, USA) and R programming language (version 3.2.3) were used for statistical analyses. Survival distributions were estimated by using Kaplan-Meier survival analysis (GraphPad Software Inc., La Jolla, CA, USA). Univariate and multivariate Cox regression analyses evaluated the hazard ratios (HRs) of different prognostic factors and were used to identify the independent prognostic factor. A two-sided $P$ value less than 0.05 was set to be statistically significant. Biological process and Kyoto Encyclopedia of Genes and Genomes (KEGG) analysis were conducted by using DAVID (the Database for Annotation,
Visualization and Integrated Discovery, https://david-d .ncifcrf.gov/home.jsp) [24, 25].

\section{Results}

3.1. FAM225B Was Dysregulated in rGBMs. We first investigated the expression pattern of FAM225B. The expression of FAM225B showed significant difference between the pGBMs and rGBMs $(P=0.0009$, Figure $1(\mathrm{a}))$. Moreover, the expression of FAM225B increased with grades of gliomas $(P=0.0436 ; P<0.0001 ; P<0.0001$, Figure $1(\mathrm{a}))$. Then, we compared the FAM225B expression level in primary and recurrent gliomas of grade II $(P=0.0568)$ and grade III $(P=0.0629$, Figure $1(\mathrm{a}))$.

The IDH1 and 1p/19q status is related to the prognosis of gliomas [26-29]. Therefore, we explored the expression pattern of FAM $225 B$ in the subtypes of rGBMs. The result showed that the expression of FAM225B enhanced in the IDH1-wild-type subtype $(P=0.0408$, Figure $1(b))$. However, no statistically significant differences were observed in the expression of FAM 225B in rGBM patients with $1 \mathrm{p} / 19 \mathrm{q}$-codeletion or $1 \mathrm{p} / 19 \mathrm{q}$-noncodeletion $(P=0.0751$, Figure $1(\mathrm{~b}))$.

3.2. FAM225B Predicted Poorer Overall Survival in rGBMs. All 109 rGBM samples were randomly divided into training set and validation set with no significant data bias. By using the median relative expression of FAM225B as the cut-off value, samples were divided into two categories including the low-expression group and high-expression group. Kaplan-Meier analysis was used to investigate the correlation between FAM225B expression and OS of rGBM patients.

We found that the OS of patients in the low-expression group were significantly longer $(P=0.0041$, Figure $2(a))$. In the training group, the OS of patients in the highexpression group were shorter than those in the lowexpression group $(P=0.0340$, Figure 2(b)). And similar 
FAM225B (rGBM)

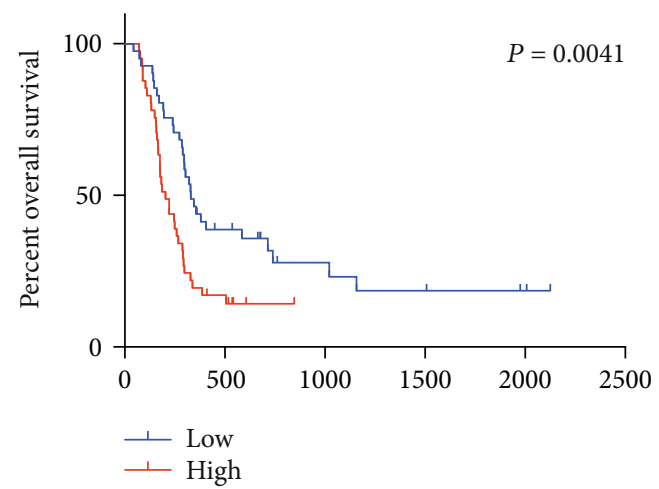

(a)
FAM225B (rGBM-training)

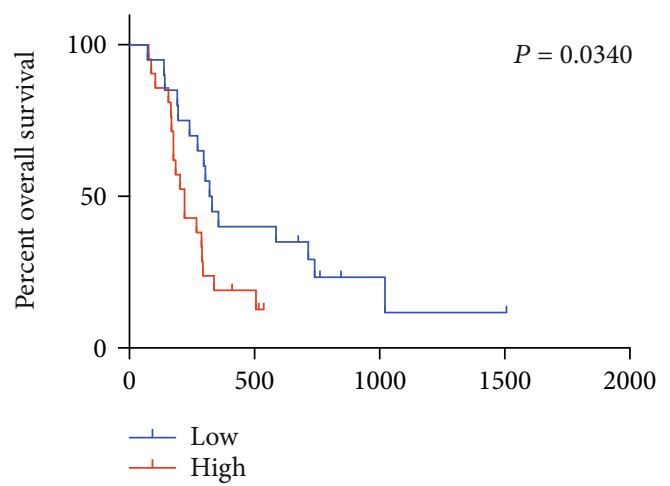

(b)

FAM225B (rGBM-validation)

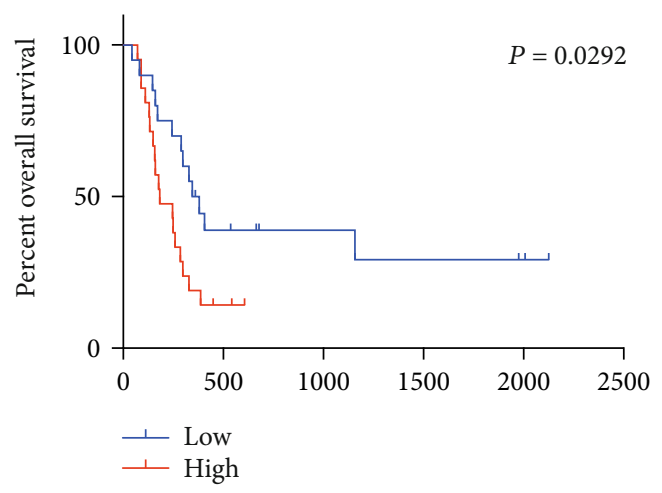

(c)

FIGURE 2: Kaplan-Meier curves of OS among rGBM patients from different groups stratified by the expression of FAM225B: (a) Kaplan-Meier curves of OS among rGBMs with different FAM225B-expressing levels (high-expression group and low-expression group); (b) Kaplan-Meier curves of OS among rGBMs with different FAM225B-expressing levels in the training set (high-expression group and low-expression group); (c). Kaplan-Meier curves of OS among rGBMs with different FAM225B-expressing levels in the validation set (high-expression group and low-expression group).

result was observed in the validation set $(P=0.0292$, Figure 2(c)).

\subsection{Associations between FAM225B and Clinicopathologic} Features. We analyzed the relationship between FAM225B expression and clinicopathologic features (Figure 3 ). The results were obtained by the univariate and multivariate Cox regression analyses (Table 1 ). The univariate Cox regression analysis found that chemotherapy $(P=0.003)$ and FAM225B $(P=0.007)$ were potential survival predictors. On the multivariate Cox regression analysis, it showed that FAM225B $(P=0.003)$ was an independent prognostic factor. $F A M 225 B$ was significantly related to the prognosis of rGBMs.

3.4. Functional Annotation of FAM225B. To explain the different prognoses of rGBMs divided by FAM225B, we extracted 902 related genes using Pearson correlation analysis (correlation value $0.4 ; P<0.001$ ). The biological process and KEGG pathway analyses of FAM 225B were performed using DAVID. It showed that major biological processes were enriched in transcription, regulation of transcription, cell division, positive regulation of migration, cell migration, etc. (Figure 4(a)). KEGG pathway analysis showed that selected genes were enriched in ubiquitin-mediated proteolysis, Wnt signaling pathway, cell cycle, focal adhesion, regulation of actin cytoskeleton, etc. (Figure 4(b)).

\section{Discussion}

Despite great efforts on the multimodal diagnosis and treatment of rGBMs, the clinical prognosis for patients remains poor due to the proliferation potential and antiapoptosis characteristics of this malignant tumor [30-32]. Therefore, understanding the expression of key factors may significantly influence the development and clinical outcome of rGBM patients. Recent studies have found that the dysregulated lncRNAs can provide insights for glioma diagnosis, prognosis, and treatment strategy [33-37].

In this study, we established a dataset of 109 rGBMs collected from the CGGA RNA sequencing dataset to explore the effect of lncRNAs on the prognosis of rGBM patients. By comparing the expression level of FAM225B between pGBMs and rGBMs, we found that FAM225B expression 
CGGA (rGBM)
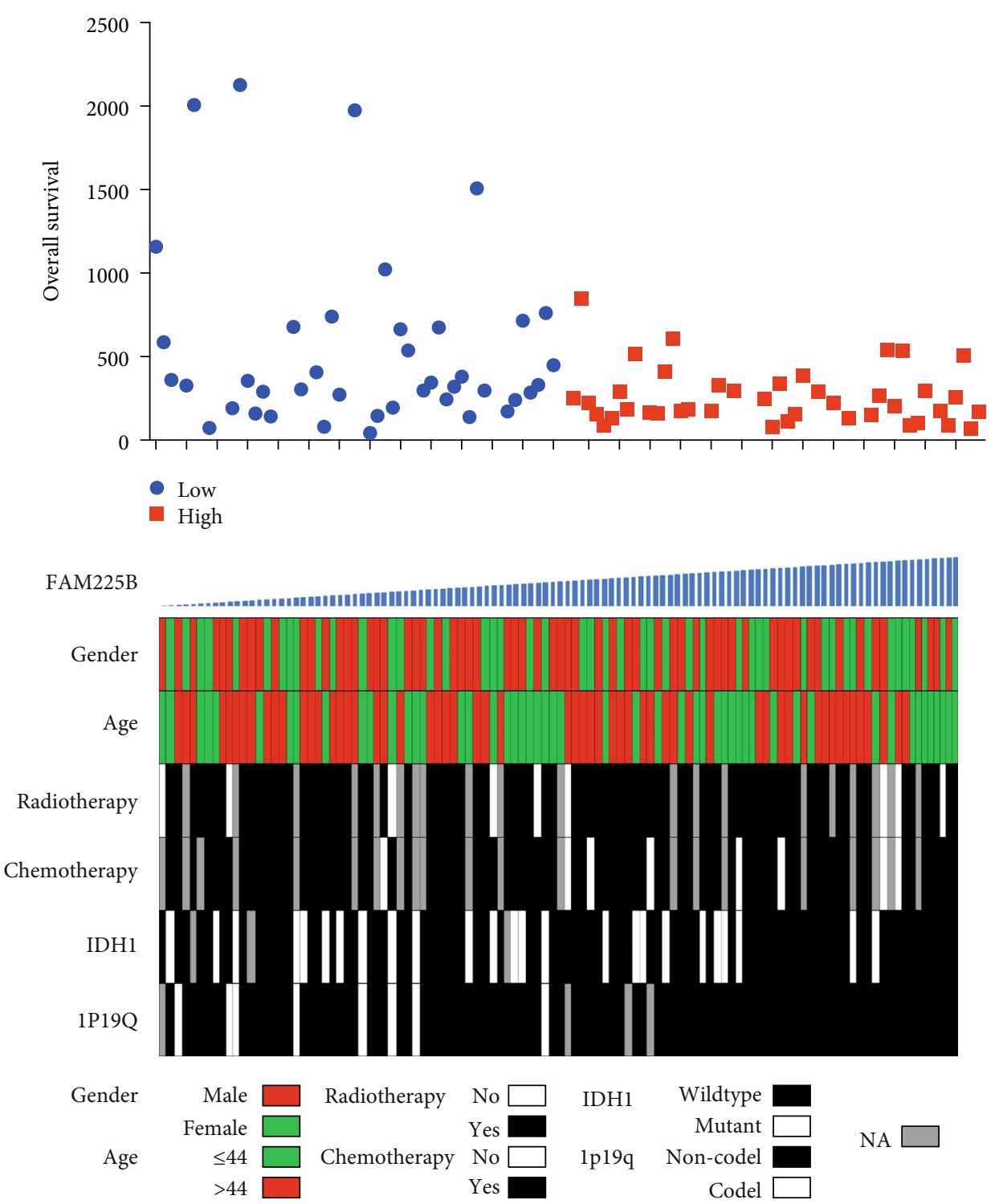

FIgURE 3: Distribution of clinicopathologic features according to the expression of FAM225B. Rows represent corresponding genes, while columns indicate corresponding patients.

TABLE 1: Univariate and multivariate Cox regression analysis of survival in rGBMs.

\begin{tabular}{lcccc}
\hline \multirow{2}{*}{ Items } & \multicolumn{2}{c}{ Univariate Cox } & \multicolumn{2}{c}{ Multivariate Cox } \\
& $p$ value & HR & $p$ value & HR \\
\hline Gender & 0.415 & 0.811 & & \\
Age & 0.286 & 1.010 & & \\
IDH1 & 0.083 & 0.645 & & \\
1p/19q & 0.100 & 0.375 & & \\
Radiotherapy & 0.102 & 1.805 & & \\
Chemotherapy & 0.003 & 0.256 & 0.003 & 0.257 \\
FAM225B & 0.007 & 1.279 & 0.003 & 1.313 \\
\hline
\end{tabular}

Gender: male, female; IDH1 status: wild type, mutant; 1p/19q status: noncodeletion, codeletion; radiotherapy: no, yes; chemotherapy: no, yes. enhanced in rGBMs $(P=0.0009)$. It indicated that FAM225B may play a key role in the recurrence process of GBMs. Then, we analyzed the expression of FAM225B in different grades of glioma, including grade II, grade III, and GBM $(P<0.0001)$. The result showed that the expression of $F A M 225 B$ was positively correlated with the grade of gliomas. This indicated that FAM225B was associated with glioma malignancy and may serve as a potential indicator for glioma grade. In addition, we explored that the expression of FAM225B enhanced in IDH1-wild type $(P=0.0408)$. Based on the data analysis above, we inferred that FAM225B can be used as a specific diagnostic marker for GBM recurrence.

We explored the correlation between FAM225B expression and survival distribution of rGBM patients. It showed 


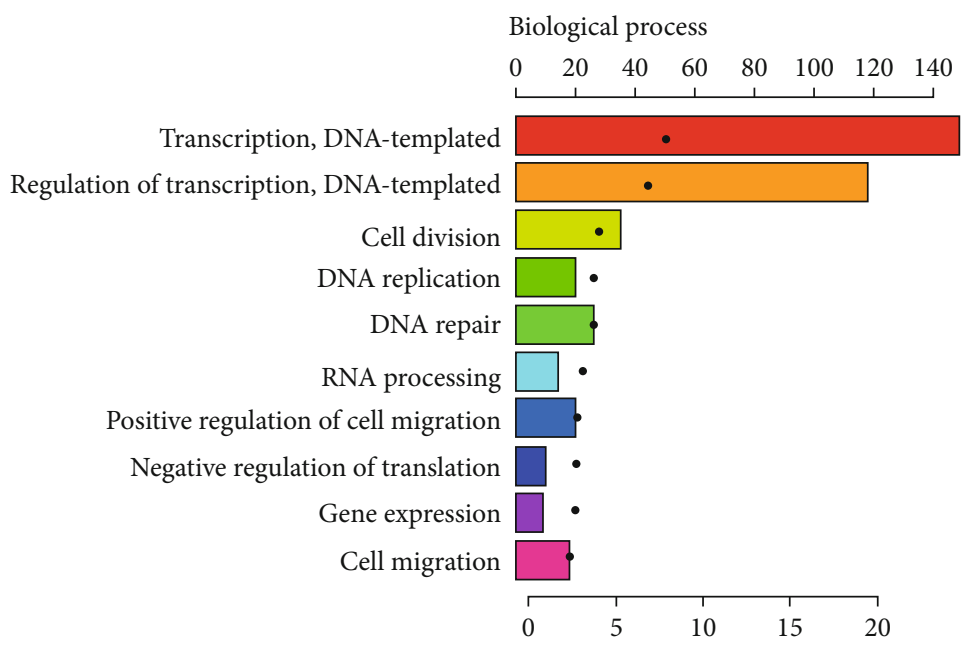

(a)

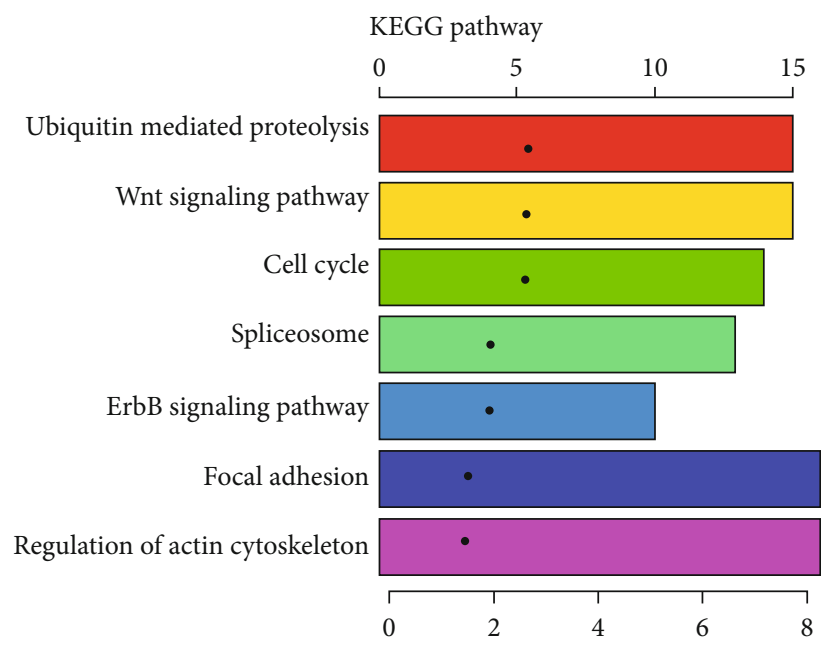

(b)

FIGURE 4: Functional annotation of FAM225B in rGBM patients: (a) biological process analysis of the related genes; (b) KEGG pathway analysis of the related genes.

that the OS decreased with the enhancement of FAM225B expression $(P=0.0041)$. FAM225B enhancement may predict the poor prognosis in rGBMs. We analyzed the relationship between FAM225B expression and clinicopathologic features. It indicated that FAM225B expression could be a significantly independent prognostic maker in rGBMs.

In our studies, functional annotation analysis showed a strong correlation between $F A M 225 B$ and transcription. It suggested that FAM225B may play an important role in rGBM through transcription and its regulation. By using the starBase website (https://starbase.sysu.edu.cn/), we found the candidate miRNAs (miR-1-3p, miR-206, and miR-205-5p) which may be correlated to FAM $225 B$. FAM 225B could competitively bind with miR-1-3p and miR-206, inducing the upregulation of AXL. It may lead to the enhancement of drug resistance and cell proliferation in glioma [38]. Furthermore, it has reported that miR-205-5p is involved in Wnt signaling, cell cycle, and focal adhesion [39], which is consistent with our KEGG findings. These processes may be associated with the recurrence of GBM. However, there are a few limitations in our study. The sample size of our dataset is still limited. Moreover, this is a retrospective study, and different treatments may have an impact on patient survival outcomes. And these findings were based on bioinformatic analysis. Prospective study is needed to clarify the mechanism of FAM225B and to further validate the clinical application in patients with rGBMs.

\section{Conclusion}

Our study found that FAM225B enhanced in rGBMs and its upregulation was related to the poor prognosis of $\mathrm{rGBM}$ patients. Therefore, our study provides a new perspective for FAM225B, as a prognosis biomarker and a potential therapeutic target for rGBMs. Further study will investigate the mechanisms of FAM225B in rGBMs. 


\section{Data Availability}

All CGGA data used in this study was available from the CGGA website (http://www.cgga.org.cn).

\section{Ethical Approval}

This study was approved by the Ethics Committee in Beijing Tiantan Hospital.

\section{Consent}

Patient informed consents were waived due to the retrospective nature of the study.

\section{Conflicts of Interest}

The authors have declared that no competing interests exist.

\section{Authors' Contributions}

Junsheng Li analyzed the results and wrote the manuscript. Qian Zhang and Peicong Ge made the statistical comparison. Chaofan Zeng and Fa Lin revised the manuscript. Wen Wang performed the gene analysis and the functional annotation analysis. Jizong Zhao designed the study. All authors have read and approved the final manuscript.

\section{Acknowledgments}

This work was supported by grants from Beijing Municipal Administration of Hospitals' Mission Plan (SML20150501); "13th Five-Year Plan" National Science and Technology supporting plan (2015BAI09B04); and Foundation of Beijing Tiantan Hospital (No. 2018-YQN-6).

\section{References}

[1] T. Zeng, L. Li, Y. Zhou, and L. Gao, "Exploring Long noncoding RNAs in glioblastoma: regulatory mechanisms and clinical potentials," International journal of genomics, vol. 2018, Article ID 2895958, 13 pages, 2018.

[2] L. Geraldo, C. Garcia, A. da Fonseca et al., "Glioblastoma therapy in the age of molecular medicine," Trends in cancer, vol. 5, no. 1, pp. 46-65, 2019.

[3] P. Tully, A. Gogos, C. Love, D. Liew, K. Drummond, and A. Morokoff, "Reoperation for recurrent glioblastoma and its association with survival benefit," Neurosurgery, vol. 79, no. 5, pp. 678-689, 2016.

[4] R. Steffens, S. Semrau, G. Lahmer et al., "Recurrent glioblastoma: who receives tumor specific treatment and how often?," Journal of Neuro-Oncology, vol. 128, no. 1, pp. 85-92, 2016.

[5] A. Krivoshapkin, A. Gaytan, N. Salim et al., "Repeat resection and intraoperative radiotherapy for malignant gliomas of the brain: a history and review of current techniques," World Neurosurgery, vol. 132, pp. 356-362, 2019.

[6] M. Weller, E. le Rhun, M. Preusser, J. Tonn, and P. Roth, "How we treat glioblastoma," ESMO open, vol. 4, article e000520, Suppl 2, 2020.
[7] D. Botros, H. Dux, C. Price, A. Khalafallah, and D. Mukherjee, "Assessing the efficacy of repeat resections in recurrent glioblastoma: a systematic review," Neurosurgical Review, 2020.

[8] J. Huang, R. Chaudhary, A. L. Cohen et al., "A multicenter phase II study of temozolomide plus disulfiram and copper for recurrent temozolomide-resistant glioblastoma," Journal of Neuro-Oncology, vol. 142, no. 3, pp. 537-544, 2019.

[9] F. Kazmi, Y. Y. Soon, Y. H. Leong, W. Y. Koh, and B. Vellayappan, "Re-irradiation for recurrent glioblastoma (GBM): a systematic review and meta-analysis," Journal of Neuro-Oncology, vol. 142, no. 1, pp. 79-90, 2019.

[10] B. Campos, L. R. Olsen, T. Urup, and H. S. Poulsen, "A comprehensive profile of recurrent glioblastoma," Oncogene, vol. 35, no. 45, pp. 5819-5825, 2016.

[11] A. O. Sasmita, Y. P. Wong, and A. P. K. Ling, "Biomarkers and therapeutic advances in glioblastoma multiforme," AsiaPacific Journal of Clinical Oncology, vol. 14, no. 1, pp. 4051, 2018.

[12] I. Ulitsky and D. P. Bartel, "lincRNAs: genomics, evolution, and mechanisms," Cell, vol. 154, no. 1, pp. 26-46, 2013.

[13] J. Li, H. Meng, Y. Bai, and K. Wang, "Regulation of lncRNA and its role in cancer metastasis," Oncology Research, vol. 23, no. 5, pp. 205-217, 2016.

[14] P. J. Batista and H. Y. Chang, "Long noncoding RNAs: cellular address codes in development and disease," Cell, vol. 152, no. 6, pp. 1298-1307, 2013.

[15] J. H. Noh, K. M. Kim, W. G. McClusky, K. Abdelmohsen, and M. Gorospe, "Cytoplasmic functions of long noncoding RNAs," Wiley interdisciplinary reviews RNA, vol. 9, no. 3, article e1471, 2018.

[16] S. U. Schmitz, P. Grote, and B. G. Herrmann, "Mechanisms of long noncoding RNA function in development and disease," Cellular and molecular life sciences, vol. 73, no. 13, pp. 24912509, 2016.

[17] M. Zhou, Z. Zhang, H. Zhao, S. Bao, L. Cheng, and J. Sun, “An immune-related six-lncRNA signature to improve prognosis prediction of glioblastoma multiforme," Molecular Neurobiology, vol. 55, no. 5, pp. 3684-3697, 2017.

[18] S. Liu, R. Mitra, M. M. Zhao et al., "The potential roles of long noncoding RNAs (IncRNA) in glioblastoma development," Molecular Cancer Therapeutics, vol. 15, no. 12, pp. 29772986, 2016.

[19] M. Li, S. Long, J. Hu, Z. Wang, C. Geng, and S. Ou, "Systematic identification of lncRNA-based prognostic biomarkers for glioblastoma," Aging, vol. 11, no. 21, pp. 9405-9423, 2019.

[20] W. X. Peng, P. Koirala, and Y. Y. Mo, "LncRNA-mediated regulation of cell signaling in cancer," Oncogene, vol. 36, no. 41, pp. 5661-5667, 2017.

[21] W. Cheng, X. Ren, C. Zhang et al., "Bioinformatic profiling identifies an immune-related risk signature for glioblastoma," Neurology, vol. 86, no. 24, pp. 2226-2234, 2016.

[22] W. Wang, Z. Zhao, F. Wu et al., "Bioinformatic analysis of gene expression and methylation regulation in glioblastoma," Journal of Neuro-Oncology, vol. 136, no. 3, pp. 495-503, 2018.

[23] J. X. Zhang, L. Han, Z. S. Bao et al., "HOTAIR, a cell cycleassociated long noncoding RNA and a strong predictor of survival, is preferentially expressed in classical and mesenchymal glioma," Neuro-Oncology, vol. 15, no. 12, pp. 1595-1603, 2013.

[24] L. Chen, Y. H. Zhang, G. Lu, T. Huang, and Y. D. Cai, “Analysis of cancer-related lncRNAs using gene ontology and 
KEGG pathways," Artificial Intelligence in Medicine, vol. 76, pp. 27-36, 2017.

[25] L. Chen, Y. H. Zhang, S. Wang, Y. Zhang, T. Huang, and Y. D. Cai, "Prediction and analysis of essential genes using the enrichments of gene ontology and KEGG pathways," PLoS One, vol. 12, no. 9, article e0184129, 2017.

[26] W. Szopa, T. A. Burley, G. Kramer-Marek, and W. Kaspera, "Diagnostic and therapeutic biomarkers in glioblastoma: current status and future perspectives," BioMed Research International, vol. 2017, Article ID 8013575, 13 pages, 2017.

[27] K. Ludwig and H. I. Kornblum, "Molecular markers in glioma," Journal of Neuro-Oncology, vol. 134, no. 3, pp. 505512, 2017.

[28] M. Rahman, J. Kresak, C. Yang et al., "Analysis of immunobiologic markers in primary and recurrent glioblastoma," Journal of Neuro-Oncology, vol. 137, no. 2, pp. 249-257, 2018.

[29] K. Aldape, G. Zadeh, S. Mansouri, G. Reifenberger, and A. von Deimling, "Glioblastoma: pathology, molecular mechanisms and markers," Acta Neuropathologica, vol. 129, no. 6, pp. 829-848, 2015.

[30] K. Seystahl, W. Wick, and M. Weller, "Therapeutic options in recurrent glioblastoma-an update," Critical Reviews in Oncology/Hematology, vol. 99, pp. 389-408, 2016.

[31] J. L. Shah, G. Li, J. L. Shaffer et al., "Stereotactic radiosurgery and hypofractionated radiotherapy for glioblastoma," Neurosurgery, vol. 82, no. 1, pp. 24-34, 2018.

[32] J. M. Frischer, C. Marosi, A. Woehrer et al., "Gamma knife radiosurgery in recurrent glioblastoma," Stereotactic and Functional Neurosurgery, vol. 94, no. 4, pp. 265-272, 2016.

[33] F. Wu, C. Zhang, J. Cai et al., "Upregulation of long noncoding RNA HOXA-AS3 promotes tumor progression and predicts poor prognosis in glioma," Oncotarget, vol. 8, no. 32, pp. 53110-53123, 2017.

[34] W. Zhao, C. Sun, and Z. Cui, “A long noncoding RNA UCA1 promotes proliferation and predicts poor prognosis in glioma," Clinical and Translational Oncology, vol. 19, no. 6, pp. 735-741, 2017.

[35] C. Y. Dong, J. Cui, D. H. Li, Q. Li, and X. Y. Hong, "HOXA10AS: a novel oncogenic long non-coding RNA in glioma," Oncology Reports, vol. 40, no. 5, pp. 2573-2583, 2018.

[36] H. Xiao, N. Ding, H. Liao et al., "Prediction of relapse and prognosis by expression levels of long noncoding RNA PEG10 in glioma patients," Medicine, vol. 98 , no. 45, article e17583, 2019.

[37] K. X. Ma, H. J. Wang, X. R. Li et al., "Long noncoding RNA MALAT1 associates with the malignant status and poor prognosis in glioma," Tumor Biology, vol. 36, no. 5, pp. 3355-3359, 2015.

[38] Y. Ma, G. Zhou, M. Li et al., "Long noncoding RNA DANCR mediates cisplatin resistance in glioma cells via activating AXL/PI3K/Akt/NF- $\kappa$ B signaling pathway," Neurochemistry International, vol. 118, pp. 233-241, 2018.

[39] B. L. Zhang, F. L. Dong, T. W. Guo, X. H. Gu, L. Y. Huang, and D. S. Gao, "MiRNAs mediate GDNF-induced proliferation and migration of glioma cells," Cellular Physiology and Biochemistry, vol. 44, no. 5, pp. 1923-1938, 2018. 